\title{
Comparación del cultivo celular de HeLa y HEp-2: Perspectivas de estudios con Chlamydia trachomatis
}

\author{
Comparison of cell culture HeLa and HEp-2: Studies prospects \\ Chlamydia trachomatis
}

Laura Camila Carrera Páez ${ }^{1-3}$, Ingrid Daniela Pirajan Quintero ${ }^{1-4}$, María Camila Urrea Suarez ${ }^{1-5}$, Martha Gómez Jiménez ${ }^{2-7}$, Luz Adriana Monroy Cano ${ }^{2-8}$, Ruth Melida Sanchez Mora ${ }^{2-6}$.

\section{Resumen}

Objetivo. Estandarizar el cultivo de células HeLa en diferentes condiciones, con el fin de utilizarlo en protocolos de infección con Chlamydia trachomatis serovar L2. Métodos. Este estudio se llevó a cabo en cuatro fases principales: 1.Viabilidad celular por la técnica de azul de tripán y su posterior observación, 2. Estandarización del cultivo de células HeLa, 3. Coloración de Giemsa, 4. Cultivo de células HEp-2. Resultados. Se determinó que la línea celular HeLa debe ser cultivada en medio DMEM, 0,1\% de L- glutamina, 10\% de SFB. Así mismo, que la coloración de Giemsa es mejor realizarla en un tiempo de 40 minutos por que se evidencia una clara definición de núcleo y citoplasma. Frente a la comparación de las dos líneas celulares se obtuvo que la línea HeLa desde el primer día muestra un crecimiento adecuado y alcanza rápidamente la confluencia esperada, en contraposición la línea HEp-2 presenta un crecimiento más lento pero alcanzando la confluencia deseada al último día.

Palabras clave. Chlamydia trachomatis, Células HeLa, Células HEp-2, Cultivo celular, infección celular

\begin{abstract}
Objective. The goal of this study was to standardize the cultivation of HeLa cells under different conditions, to be used in Chlamydia trachomatis serovar L2 infection's protocols. Methods. This study was conducted in four phases: 1. Cell Viabilidad by trypan blue technique and his subsequent remark, 2. Standardization HeLa cell culture, 3. Giemsa, 4. Cultivation of Hep-2 cells. Results. As a result of standardization it is determined that the HeLa cell line should be cultured in DMEM, 0.1\% L-glutamine, 10\% FBS.It was determined that the Giemsa perform better over time of 40 minutes that a clear definition of nucleus and cytoplasm is evident. Comparing against both cell lines HeLa was obtained that the line from day growing well and quickly reaches the expected confluence, the opposed line HEp-2 has a slower growth but achieving the desired confluence the last day .
\end{abstract}

Key words: Chlamydia trachomatis, HeLa cells, HEp-2 cells, cell culture, cell infection

1. Estudiantes Universidad Colegio Mayor de Cundinamarca

2. Docentes Universidad Colegio Mayor de Cundinamarca

ORCID: ${ }^{3} 0000-0002-9714-9471,{ }^{4} 0000-0002-0641-6395,{ }^{5} 0000-0003-4480-4257,{ }^{6} 0000-0002-0572-8418,{ }^{7} 0000-0003-0772-3909,{ }^{8} 0000-0001-5901$

Correspondencia: laurapaez_21315@hotmail.com

Recibido: 19/02/2015 Aceptado: 15/05/2012 


\section{Introducción}

Chlamydia es una bacteria Gram negativa, patógena intracelular obligada (1) capaz de infectar células epiteliales del tracto urogenital y la conjuntiva (2). Comparte un antígeno común específico de género y tiene un antígeno específico de especie que corresponde a la proteína de membrana externa MOMP (Proteína mayor de membrana externa). Este microorganismo tiene dos biotipos: tracoma y LGV (linfogranuloma venéreo) (3), que a su vez se dividen en varios serotipos, encontrados en una amplia variedad de enfermedades asintomáticas de importancia clínica y en salud pública, siendo el biotipo LGV el principal causante del linfogranuloma venéreo (1-6).

Es responsable de afectar a 90 millones de personas por año y según la OMS (Organización Mundial de la Salud) es uno de los patógenos que más se ve asociado a enfermedades de este tipo, afecta tanto a hombres como mujeres, causando infertilidad en ambos y su adquisición está relacionada con algunos factores de riesgo (3).

El linfogranuloma venéreo (LGV) puede cursar de manera asintomática casi en un $70 \%$ de las mujeres y en hombres entre un $30-50 \%$ por lo que su diagnóstico es más complicado. El tracoma puede causar ceguera y afectar a poblaciones indígenas vulnerables en edad escolar, aproximadamente es responsable de 1 millón de casos en América Latina (8). La prevalencia de la infección causada por CT es mayor en jóvenes que ya han iniciado su vida sexual entre las edades de a 20 a 24 años (9).

Las infecciones causadas por CT cursan de manera asintomática, por esta razón este microorganismo se ha convertido en un problema de salud pública y se hace necesario reducir la prevalencia de este, pero los programas de control epidemiológico se ven obstaculizados ya que para su diagnóstico no se disponen de técnicas de referencia, por lo que para realizar éste se continúan usando los cultivos de diferentes líneas celulares, siendo estos de difícil mantenimiento en el laboratorio de rutina(10).

La línea celular HeLa corresponde a células de adenocarcinoma cervical humano, las cuales pueden considerarse como la "primera línea de células humanas inmortales”, estas son adecuadas para la investigación ya que tienen un crecimiento muy rápido y agresivo, además de que poseen resistencia a la apoptosis(11). Esta línea celular ha servido por más de cincuenta años como fuente de investigación en varios campos de la medicina (11).

La línea celular Hep-2 proviene de carcinomas epidermoides laríngeos humanos, fue descrito por primera vez en 1952 y en 1954 cuando se inoculó en ratas jóvenes, esta línea es metabólicamente activa lo que favorece el cultivo de la misma y su posterior infección (12).

El objetivo de este proyecto fue estandarizar un protocolo de cultivo de células HeLa, que será comparado con el protocolo de la línea celular HEp-2 con el fin de determinar cuál de estos es el más adecuado, para realizar la infección con cepas de CT serovariedad L2. Lo que permitirá tener un modelo para el estudio básico de este microorganismo, con la finalidad de ser probado con péptidos antimicrobianos o en modelos inmunológicos. Los resultados de los cultivos podrán ser observados en un microscopio invertido y posteriormente con tinción de Giemsa para determinar si la morfología celular se mantuvo y saber entonces si es adecuada para la infección. 


\section{Materiales y métodos}

\section{Viabilidad celular por la técnica de azul de tripan}

Antes de realizar los diferentes cultivos se determinó la viabilidad por el método de exclusión con azul de tripan. Lo primero es la obtención del pellet del cultivo celular, este fue re-suspendido en $1 \mathrm{ml}$ de medio sin SFB (suero fetal bovino), de esta mezcla se toman $20 \mu \mathrm{L}$ y se le adicionan $20 \mu \mathrm{L}$ de azul de tripán; posterior a esto se mezcla bien y se realiza un montaje con $10 \mathrm{uL}$ en cada retículo de la cámara de Neubauer, el paso final es observar en el microscopio óptico, realizando el conteo de los 4 cuadrantes de las esquinas de ambos retículos para determinar el número total de células vivas (células sin teñirse) y células muertas $(13,14)$. Figura 1 .

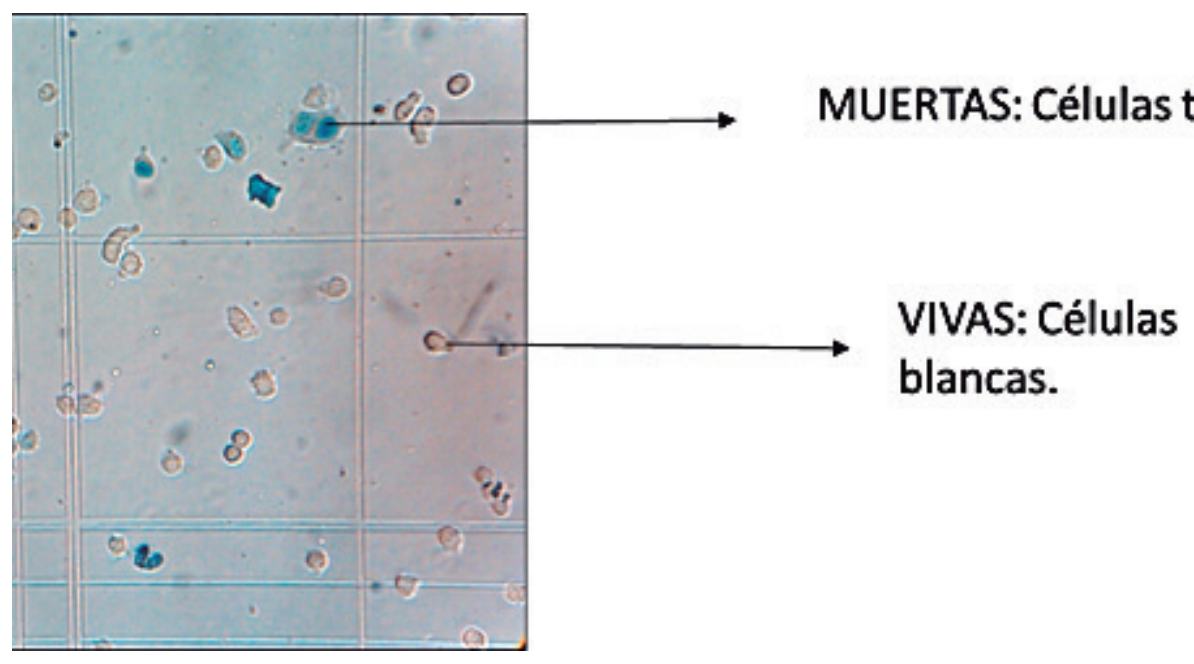

Figura 1. Determinación de viabilidad celular por la técnica de azul tripán.

Se observan células vivas y muertas obtenidas del pellet después de la descongelación de un crio vial de células HeLa.

\section{Estandarización del cultivo de células HeLa}

Para ajustar las condiciones del cultivo se descongelaron los crioviales y se procedió a realizar tres ensayos: en el primero se cultivó las células usando dos medios diferentes (DMEM y DMEM+F12 en relación 1:1) con SFB 10\% y $1 \%$ de antibiótico, el segundo ensayo se realizó usando el medio DMEM, con diferentes concentraciones de L-glutamina, en el primero se usó $0.1 \%$ y en el segundo se usó $0.05 \%$. Para finalizar se hace un ensayo variando las concentraciones de SFB al 5\% vs 10\% usando el medio DMEM y la concentración de L-glutamina establecida como la ideal, para finalizar se realizan varios ensayos con las condiciones previamente estandarizadas, todos los ensayos fueron incubados a una atmosfera de $5 \%$ de $\mathrm{CO} 2,37^{\circ} \mathrm{C}$ por 72 horas.

\section{Coloración de Giemsa}

Finalmente se estandarizó el tiempo ideal para la realización de la coloración de Giemsa, en donde se pudo observar la morfología celular de una manera más detallada, se inicia por la elaboración de los colorantes necesarios para dicha coloración. Para el colorante de Giemsa se debe preparar la solución amortiguadora y la solución 
madre, Tabla 1, posterior a esto se mezclan 72 $\mathrm{ml}$ de solución A con $28 \mathrm{ml}$ de solución $\mathrm{B}$, luego se afora a $1 \mathrm{~L}$ con agua destilada y a esta nueva mezcla se le ajusta el $\mathrm{pH}$ en 7.2 y finalmente se filtra. La coloración se realizó cultivando las células sobre una laminilla de vidrio estéril. Una vez incubadas las células se lavaron con solución salina estéril, retirando los posibles detritos, se fijan con metanol concentrado durante 15 minutos, luego de esto se agregan 3 o 4 gotas de la solución de trabajo (14), se colocan a exposición durante dos tiempos (40 minutos y 60 minutos) y posterior a esto se observan en el microscopio óptico (40X y 100X).

Tabla 1. Coloración de Giemsa

\section{Colorante de Giemsa pH 7.2 (14)}

\begin{tabular}{lcc} 
Solución madre & Solución amortiguadora & $(\mathrm{pH} \mathrm{7.2)}$ \\
\cline { 1 - 2 } Colorante $0,5 \mathrm{gr}$ & Solución A & Solución B \\
Metanol libre de acetona $33 \mathrm{ml}$ & $9,5 \mathrm{gr} \mathrm{Na} 2 \mathrm{HPO} 4$ & $9,2 \mathrm{gr} \mathrm{NaH2PO4}$ \\
Glicerol $33 \mathrm{ml}$ & $\mathrm{H} 2 \mathrm{O}$ destilada $1000 \mathrm{ml}$ & $\mathrm{H} 2 \mathrm{O}$ destilada $1000 \mathrm{ml}$
\end{tabular}

\section{Cultivo de células HEp-2}

Se realizó el cultivo de células HEp-2 bajo los parámetros establecidos por Juntinico et al en el 2013 (14) en el grupo de Biotecnología y Genética UCMC de la Universidad Colegio Mayor de Cundinamarca.

\section{Resultados}

Dentro de este proyecto se realizaron diferentes ensayos con las condiciones necesarias para la estandarización del cultivo de la línea celular HeLa, dentro de estas variables se tomaron la concentración necesaria de SFB, L- glutamina y el medio ideal para el crecimiento de las mismas. Teniendo la estandarización del cultivo de las células HeLa se comparó con el protocolo de cultivo de la línea celular HEp-2, para determinar cuál de estas dos líneas es la más indicada para realizar posteriores estudios de infección con Chlamydia trachomatis serovariedad L2.
Las condiciones ajustadas en cada ensayo son observadas a diario en microscopio invertido, observando así las diferentes confluencias y estableciendo cuál de los estudios era el más efectivo. Para iniciar cada ensayo se determinó la viabilidad por el método de exclusión con azul de tripan.

\section{Viabilidad celular}

Para la determinación de viabilidad celular se usaron diferentes métodos dentro de los cuales se encuentra el de exclusión por azul de tripan, llamado así debido a que las células vivas (células sin teñir) excluyen el colorante debido a la resistencia de su membrana; por el contrario, las células muertas tienen una membrana desestabilizada, por tanto permiten el ingreso del colorante, obteniéndose como resultado células teñidas de color azul (13), como se evidencia en la Figura 1.

Todos los ensayos fueron realizados con crio viales que presentaron una viabilidad del $85-97 \%$. 
Para desarrollar diferentes investigaciones es importante obtener una viabilidad mínima del 85\%.

\section{Estandarización del cultivo de células HeLa}

En la estandarización del medio a usar para el cultivo de células HeLa, se determinó por literatura que se emplean una variedad de medios, entre los que se encuentran MEM, RPMI, DMEM entre otros $(9,10)$ de estos se seleccionó el DMEM y DMEM: F12 medios utilizados por Juntinico et al, para la estandarización de células HEp-2 (14); estos ensayos se realizan en placas de 6 pozos y se monitorearon durante 3 días.
El crecimiento de las células HeLa en los medios fue comparado teniendo en cuenta la confluencia obtenida desde las 24 horas hasta a las 72 horas después de haber iniciado el cultivo a las 0 horas. Posterior a las 72 horas se logró evidenciar que en el cultivo con el medio DMEM hay un crecimiento óptimo y constante obteniéndose una confluencia promedio del $95 \%$. Por otro lado en el medio DMEM:F12 se observó crecimiento constante a las 24 y 48 horas, pero más lento en comparación con el crecimiento en DMEM, sin embargo a las 72 horas se observa un crecimiento aumentado llegando a obtener una confluencia promedio del $88 \%$, Figura 2.

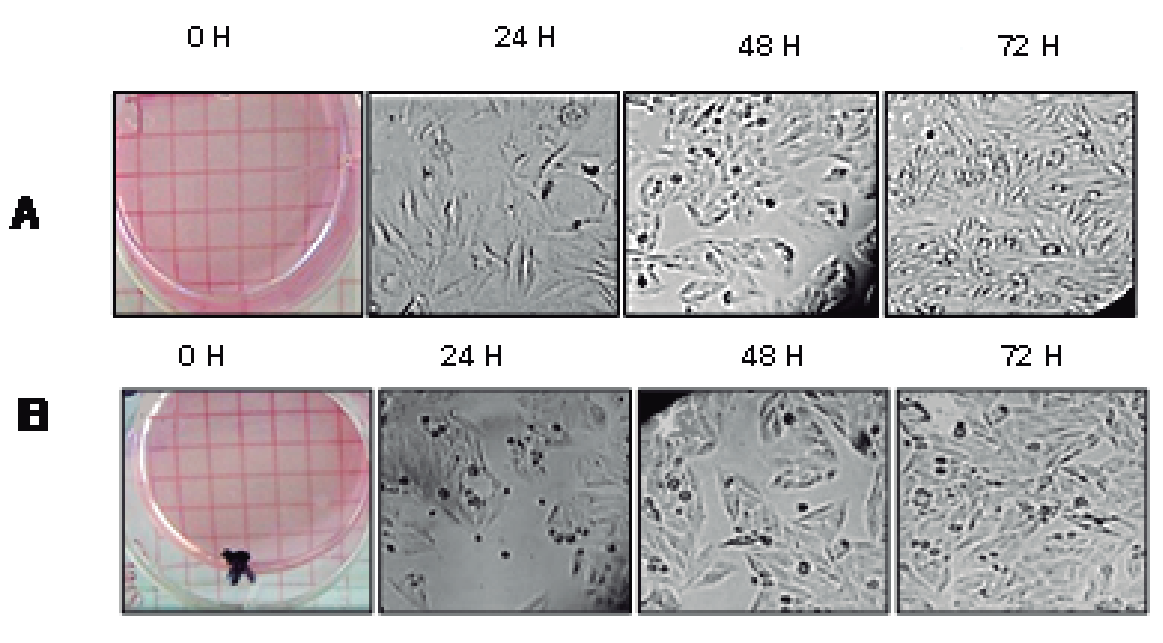

\section{c CUANTIFICACION DE LAS CONFLUENCIAS CON DOS MEDIOS DIFERENTES}

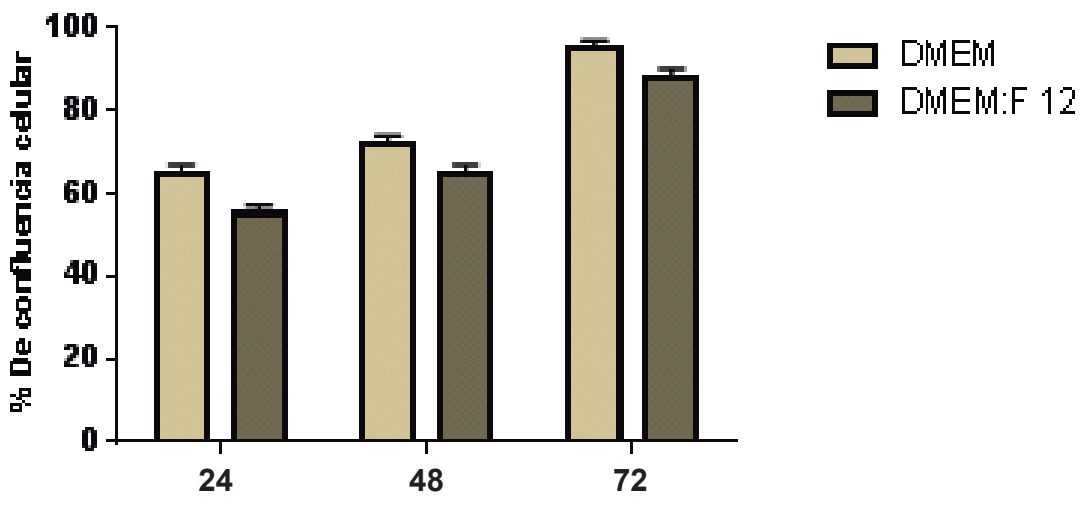

\section{Tiempo de cultivo en horas}

Figura 2. Determinación de medio para cultivo HeLa. A. Ensayo con medio DMEM. B. Ensayo con medio DMEM: F12 C. Comparación de los dos medios DMEM y DMEM: F12 
Por lo que se sugiere que el medio DMEM aporta mejores condiciones para alcanzar la confluencia necesaria durante los tres días del cultivo condición importante durante la infección de las líneas celulares con Chlamydia trachomatis.

Determinación de la concentración de L-glutamina para el cultivo de línea celular HeLa

La L- glutamina es un aminoácido, que sirve como enriquecedor del medio ya que le brinda nutrientes requeridos para aumentar el cre- cimiento y así lograr la adhesión celular, para llegar a la confluencia necesaria (15).

En la Figura 3 se determina el crecimiento de las células HeLa en dos diferentes concentraciones de L-glutamina versus la confluencia observada, se puede evidenciar que en una concentración de $0,1 \%$ las células presentan un crecimiento continuo y confluencias más altas en menor tiempo, con presencia de mayor cantidad de células adheridas al pozo, en comparación con las células que fueron cultivadas con $0,05 \%$ que no alcanzan la confluencia necesaria.
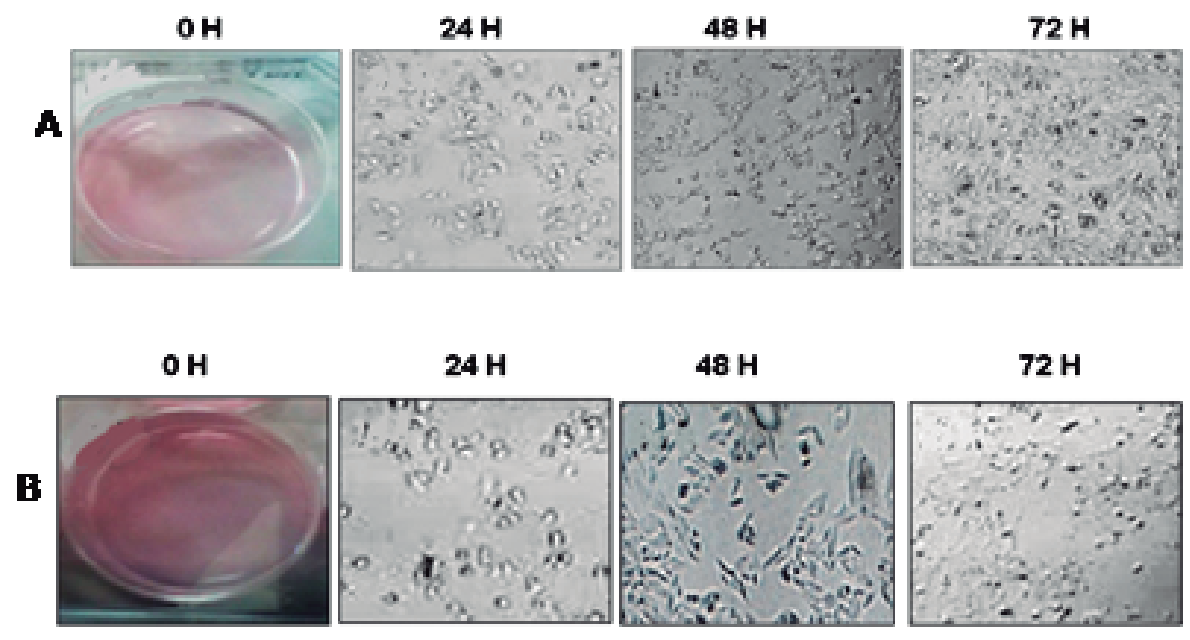

\section{C}

\section{CULTNO DE CELULAS HeLa CON DIFERENTES PORCENTAJES DE L- GLUTAMINA}

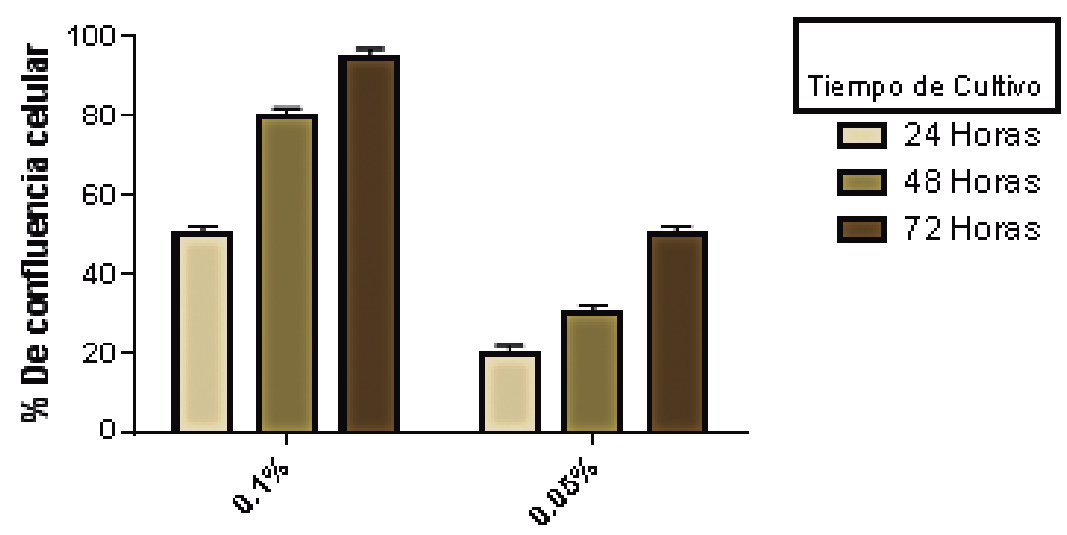

$\%$ DE L- Glutamina

Figura 3. Cultivo de células HeLa con diferentes concentraciones de L-glutamina. A. Observación con una concentración de $0,1 \%$ de L-glutamina B. Observación con una concentración de $0.05 \%$.C. Comparación de las dos concentraciones de L-Glutamina para el cultivo de la línea celular HeLa. 


\section{Estandarización del suero fetal bovino}

El Suero Fetal Bovino (SFB) aporta factores de crecimiento como hormonas, minerales, lípidos $\mathrm{y}$ otros micronutrientes, que al ser empleados en concentraciones apropiadas en el medio de cultivo, suplen satisfactoriamente los requerimientos metabólicos que garantizan la proliferación y adhesión celular, siendo uno de los productos biológicos más empleados en el mantenimiento de cultivos celulares (16). El ensayo para SFB se realiza con dos concentraciones diferentes: el primero con $5 \%$ y el segundo con $10 \%$.El desarrollo de la línea celular en función del porcentaje de suero fetal bovino agregado se puede observar en la Figura 4.

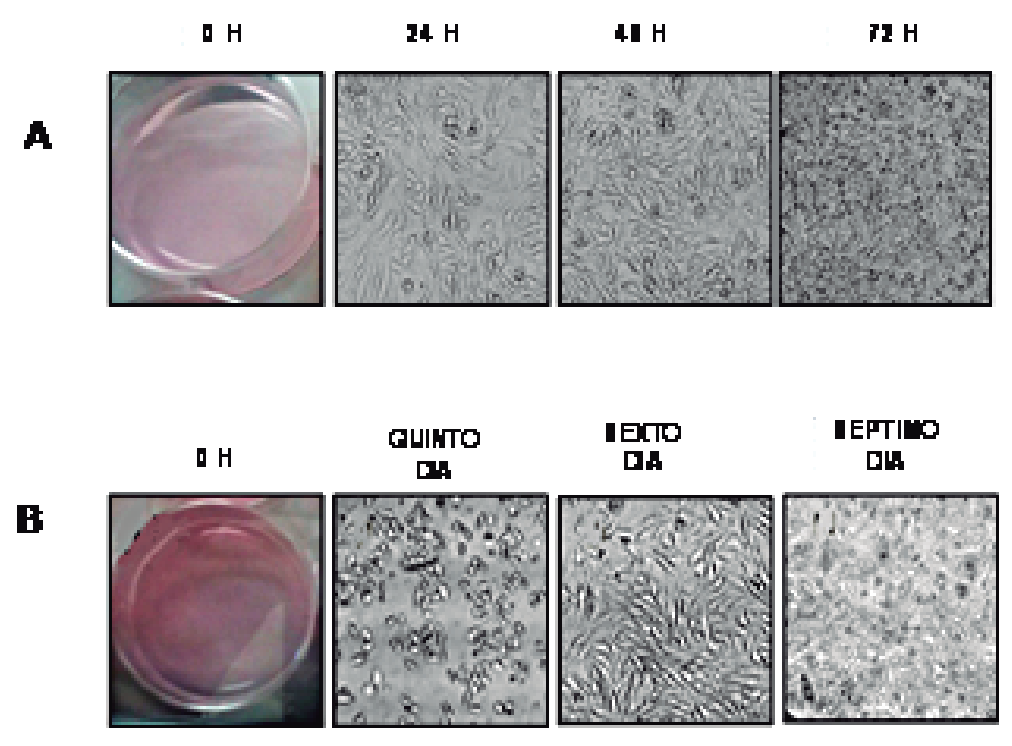

\section{COMPARACION DE SUERO FETAL BOVNO}

C

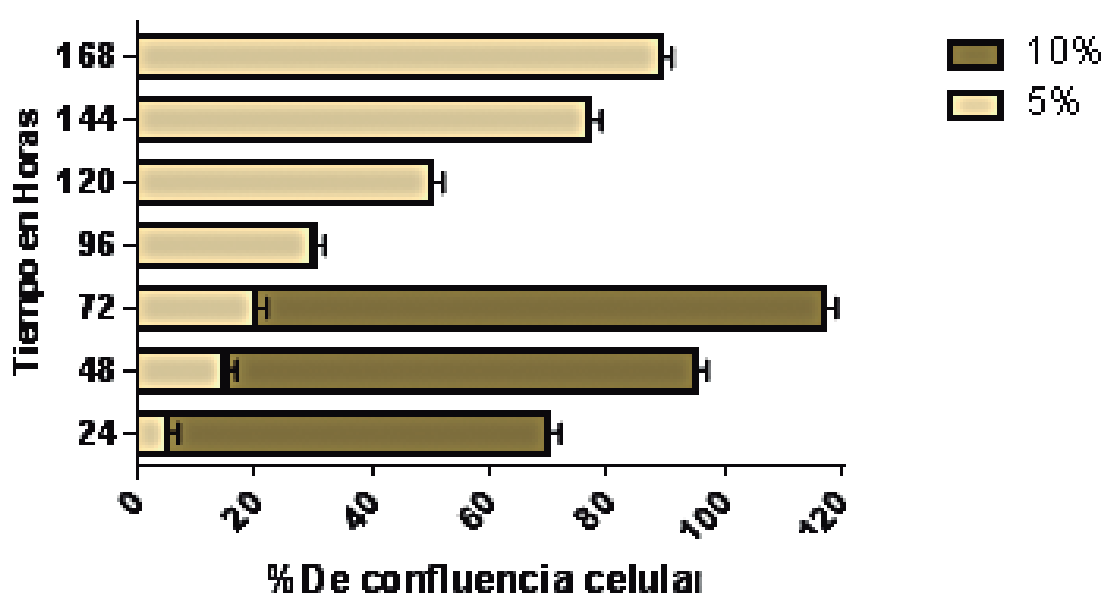

Figura 4. Comparación de diferentes concentraciones de SFB. El crecimiento con $10 \%$ de SFB es más rápido y muestra confluencias más altas que en comparación con 5\% de SFB.A. Cultivo celular con 10\% SFB. B. Cultivo celular con $5 \%$ SFB a partir del quinto día. C. Comparación de la concentración del SFB en las dos concentraciones estudiadas. 
El crecimiento con SFB al 5\% es más lento y hace que las células se encuentren sometidas a estrés, por lo que se disminuye la confluencia en los primeros días, por lo cual es necesario extender el tiempo del cultivo lo que aumenta el riesgo de contaminación, en contra posición se determina que las células cultivadas con $10 \%$ de SFB tienen un crecimiento rápido que desde el primer día muestra células con morfología definida que al tercer día alcanzan la confluencia deseada para los ensayos de infección.

Después de realizar los diferentes ensayos previos para la determinación de L-glutamina, SFB y el medio a usar, se procedió a realizar los cultivos con las condiciones obtenidas, unificando así todas las variables para la estandarización del cultivo de la línea celular HeLa. Como resultados de estandarización se determinó que la línea celular HeLa debe ser cultivada en medio DMEM, 0,1\% de L- glutamina, 10\% de SFB.

\section{Coloración de Giemsa}

Para determinar el porcentaje de infección de la línea celular HeLa con Chlamydia trachomatis serovariedad L2 fué necesario estandarizar la coloración de giemsa optima que permitió evidenciar las inclusiones de los cuerpos elementales y reticulares, para esto se estandarizó el tiempo de la coloración de Giemsa sobre la línea celular HeLa.

La coloración de Giemsa se basa en la combinación de distintos colorantes, el azul de metileno como tinte básico y la eosina tinte acido, el azul de metileno es un colorante metacromatico que da como resultado un color púrpura-azulado (17). El buen resultado de la tinción es influido por el $\mathrm{pH}$ de la solución madre, la solución amortiguadora, el tiempo de tinción y la fijación (18).
Las células fueron cultivadas y fijadas a la laminilla de vidrio con metanol concentrado y la solución de trabajo en un $\mathrm{pH}$ de 7.5. Se colorearon durante 40 minutos y 60 minutos observando que a los 40 minutos se evidencia una clara definición de núcleo y el citoplasma se ve sin precipitantes de colorante, lo cual nos permitirá realizar una buena observación en el momento de la infección, Figura 5.

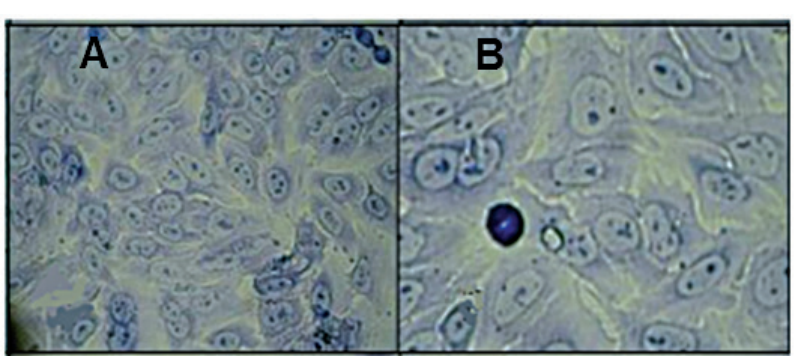

\section{$\begin{array}{ll}\text { A. } 40 \text { minutos } & \text { B. } 60 \text { minutos }\end{array}$}

Figura 5. Coloración de las células HeLa: Se observan células HeLa no infectadas, en microscopio óptico (40X), coloreadas con solución de trabajo; puesta en contacto con las células fijadas en dos tiempos.

\section{Cultivo de la linea celular HEp-2}

La línea celular HEp-2, es utilizada para diferentes estudios clínicos y de infección, siendo de gran ayuda en la creación de vacunas y péptidos antimicrobianos. Por esta razón se decide realizar el cultivo de estas células según el protocolo estandarizado por Juntinico et al en la UCMC en el año 2013 (14).

Al realizar el cultivo se puede observar que el porcentaje de confluencia de las células HEp-2 es de $90 \%$, determinando que dicha concentración es ideal para el crecimiento y multiplicación del cultivo celular logrando alcanzar la confluencia necesaria en 72 horas, Figura 6. Comparación de línea celular HeLa versus línea celular HEp-2. 
A
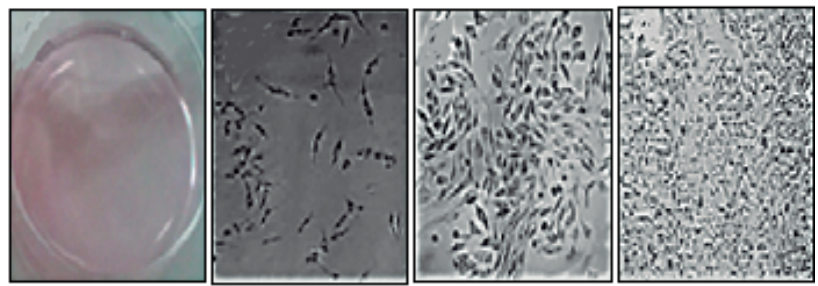

B PROMEDIO DE CONFLUENCIAS PRESENTADAS EN LOS

CULTIYOS DE CELULAS HEP.2

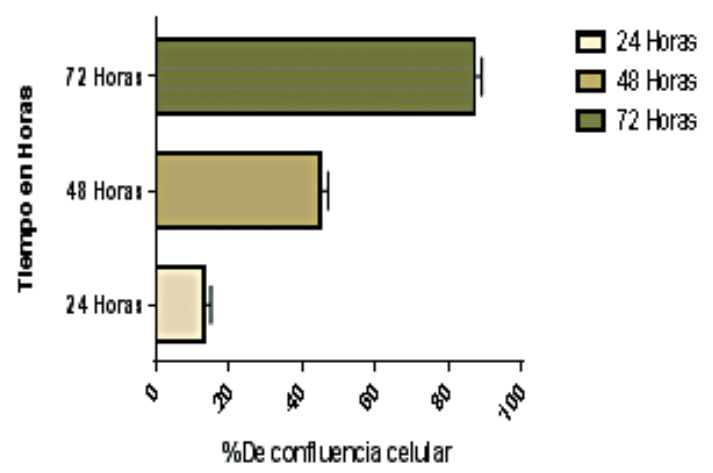

Figura 6. Cultivo de células HEp-2 en medio completo: A. (DMEM + F12, suplementado con 10\% de suero fetal bovino, $1 \%$ de antibiótico/antimicótico y $0.1 \%$ de L- glutamina), en una atmosfera de $5 \%$ de $\mathrm{CO}_{2}$ a $37^{\circ} \mathrm{C}$. B. Porcentaje de confluencia cultivo de HEp-2

Posterior a la estandarización del protocolo de cultivo de células HeLa, se procede a comparar las dos líneas celulares en estudio Tabla 2.

Tabla 2. Comparación de la línea celular HeLa Versus HEp-2

\begin{tabular}{lcc} 
& HeLa & HEp-2 \\
\hline Confluencia & $90-97 \%$ & $85-90 \%$ \\
\hline Tiempo & 1 a 3 días & 1 a 3 días \\
\hline L-Glutamina & $0,1 \%$ & $0,1 \%$ \\
\hline Detritos & No hay presencia & Aumentados \\
SFB & $10 \%$ & $10 \%$ \\
\hline Antibiótico & $1 \%$ & $1 \%$ (Mezcla antibióticos) \\
Confluencia inicial (1er día) & Ampicilina $/$ tetraciclina / & $10-15 \%$ \\
\hline Medio & antimicótico & DMEM:12 \\
\hline Costos & $65-75 \%$ & Altos \\
\hline
\end{tabular}


En la Figura 7 se muestra el promedio de los ensayos realizados con las condiciones estandarizadas en la línea celular HeLa vs HEp-2, se puede observar como la línea HeLa desde el primer día muestra un crecimiento adecuado y alcanza rápidamente la confluencia esperada, en contraposición la línea HEp-2 presenta un crecimiento más lento pero alcanzando la confluencia deseada al último día.

\section{linea HEp-2 vs la linea HeLa}

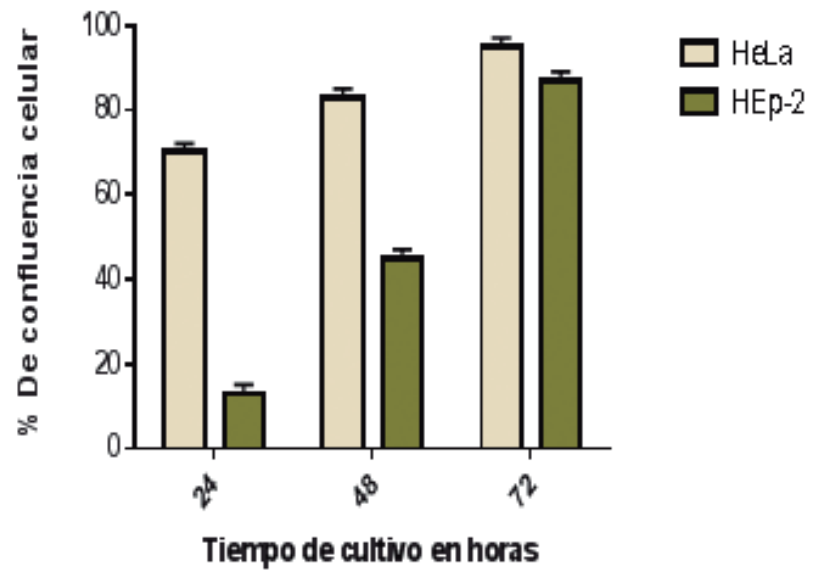

Figura 7. Confluencia celular de la línea HEp-2 vs la línea HeLa. Confluencias en el cultivo de la a línea celular HeLa con respecto a la HEp-2.

\section{Discusión}

La línea celular HeLa fue establecida como base de estudio para encontrar la cura contra el cáncer procurando que células humanas se multiplicaran fuera de un organismo de manera in vitro. A partir de este momento esta línea celular se convirtió en el blanco de diferentes estudios entre los cuales encontramos ensayos de infección (11). En este estudio se buscó optimizar el cultivo de la línea celular HeLa con el fin de infectarlas con CT. Esto se ha utilizado en otros estudios, como el realizado por Stoker et al (1958), en el Departamento de Patología de la Universidad de Cambridge, donde usaron células HeLa como modelo para determinar el proceso de infección del Herpes virus.
La propagación viral fue monitoreada y encontraron que a las 12 horas de propagación las células ya estaban infectadas (19). En este estudio se recomienda infectar las células HeLa al llegar a la confluencia deseada entre $85-95 \%$, la cual es alcanzada aproximadamente a las 72 horas.

La determinación de las características específicas de crecimiento para la línea celular HeLa se ha realizado a través de diversos estudios, desde 1958 se empezaron a cultivar con Medio Eagle suplementado con $10 \%$ de suero humano, a una atmosfera de $5 \% \mathrm{CO}_{2}$ a $37^{\circ} \mathrm{C}(20)$, posteriormente en 1982 se cultivaron en medio RPMI con las mismas condiciones de temperatura y atmosfera, pero con suero fetal bovino (SFB), empleando $2 \mathrm{mM}$ L-glutamina, $100 \mathrm{U} /$ $\mathrm{ml}$ penicilina, como suplementos adicionales $\mathrm{y}$ para la disminución de contaminación microbiana (21), gracias a estos y otros estudios se pudo determinar que las condiciones óptimas de mantenimiento son $5 \%$ de $\mathrm{CO}_{2}$ y $37^{\circ} \mathrm{C}$. Para lograr la adherencia ideal de la monocapa a partir de estas referencias se determinó que es indispensable suplementar al medio con SFB al $10 \%$ y no al $5 \%$ como se recomienda en muchos cultivos celulares (22), ya que este aporta factores que ayudan la adhesión celular y es una condición importante para la infección de dichas células; como se ha visto en otros estudios los cuales han usado para el cultivo tanto de HeLa como de otros tipos de células.

Por ejemplo el estudio realizado por Yuta et al en el 2013 en donde cultivaron distintas líneas celulares, como células del mesotelioma HGF-1 y MSTO suplementándolos con 10\% de SFB (23), otro estudio es el realizado por Hivanand $e t$ al, publicado en el 2014 donde cultivaron células de fibroblastos de pulmón y HeLa-229 (24), suplementándolas con SFB al $10 \%$, cabe resaltar que durante los estudios comparados se obtuvieron porcentajes de confluencia similares a los encontrados en este proyecto que oscilan entre el 80 y $97 \%$. 
Por otra parte es muy importante efectuar un suplemento con L-glutamina, que aunque se encuentra en el medio es necesaria su adición ya que por su fácil degradación se pierde muy rápido con el tiempo, como lo hizo Wong et al en mayo del 2014 (25), permitiendo así que se logre la confluencia deseada. El uso de L-Glutamina se puede evidenciar en estudios como el realizado por Takanaka et al donde la usan para enriquecer el medio y poder obtener cultivos de la línea celular HeLa y realizar marcaje células vivas con sustrato fluorescente (26).

En el estudio realizado por Juntinico et al en el 2013 (14), con células HEp-2 que fueron cultivadas con medio DMEM: F12 y la adición externa de L-Glutamina se produjo un aumento en la presencia de detritos. En este trabajo la línea celular HeLa fue cultivada en medio DMEM y del mismo modo la L-Glutamina fue adicionada externamente como ayuda para la suplementación del medio ya que es un aminoácido imprescindible para las células $(24,27)$, ayudando a la formación de la monocapa de una manera más eficiente, sin generar detritos y en menor tiempo como se observa en la Figura 3C. El medio DMEM es un medio que no contiene L-Glutamina (28) al contrario del medio F12 el cual contiene $146 \mathrm{mg} / \mathrm{L}$ de glutamina (23), por lo cual se le adicionó de manera externa, determinando que la concentracion $0,1 \%$ es la ideal de Glutamina , ya que esta genera un crecimiento más continuo obteniendo una confluencia más alta, entre el $50 \%$ en las primeras horas y hasta un $93 \%$ al terminar el cultivo, Figura 3A. Ensayos con concentraciones menores de L-Glutamina (29), no generaron la confluencia deseada en un tiempo estimado de 3 días, Figura $3 \mathrm{~B}$, para así posterior a esto poder realizar la infección con $C T$.

Otro parámetro importante es la visualización de las células HeLa mediante la técnica de coloración de Giemsa protocolo estandarizado por Juntinico et al (14). Esta coloración permite evidenciar la morfología celular de una mejor manera, ya que resalta en la célula organelos que no se pueden ver a simple vista o de forma más clara en el microscopio de luz invertida, también en un futuro cuando se haga la infección con $C T$, permitirá evidenciar los cuerpos elementales(30). Esta coloración se realiza en esta investigación con la finalidad de determinar cuál de los dos tiempos propuesto es más adecuado, llegando a la conclusión que 40 minutos es ideal ya que no genera precipitados de colorante y permite observar la morfología celular de manera adecuada.

En muchos estudios la línea celular HeLa se cultiva y mantiene en diferentes medios como lo muestra Himeda et al, quienes usaron medio MEM y posteriormente las infectaron y observaron la cinética del virus SAFV-3 (31) o como Wang et al, que mantuvieron las células en medio RPMI 1640 para después observar como la invasión del virus del tabaco influenciaba a la célula hospedadora a nivel del retículo endoplasmatico (32). En este estudio se ejecutó el ensayo de cultivo de células HeLa con dos medios: primero con DMEM obteniéndose la confluencia deseada a las 72 horas, Figura 2A, y segundo con una combinación de medio DMEM: F12 en relación 1:1 dando como resultado una confluencia menor, Figura 2B, demostrando que no es necesario el uso del medio F12 para el cultivo de esta línea celular HeLa, lo cual disminuiría los costos de cultivo celular.

La línea celular HEp-2 (células epidermoides de cáncer laríngeo) se estableció en 1952 (28) un año después del descubrimiento de la línea celular HeLa, donde a partir de allí se comenzaron a realizar diversos estudios con diferentes medios y diferentes condiciones para establecer la forma adecuada de crecimiento. Uno de los últimos estudios es el realizado por Juntinico et al, donde realizaron un cultivo usando la combinación de medio DMEM: F12 para mantener células HEp-2 (13) y Pajaniradje et al, donde las mantuvieron en medio DMEM suplementado con $10 \% \mathrm{SFB}$, a $37^{\circ} \mathrm{C}$ en una incubadora humificada con una atmosfera de $5 \% \mathrm{CO}_{2}$ y $95 \%$ aire (29). 
En Colombia se han elaborado algunas investigaciones con líneas celulares las implementadas en protocolos de infección, como el desarrollado por Arévalo et al en el 2011 donde estudiaron la relación que tenían los péptidos sintéticos de dos proteínas que se encuentran asociadas a la invasión de esporozoitos con las líneas celulares HeLa y HepG2 (30). Otro estudio realizado con HEp-2 es el protocolo realizado por Juntinico et al en 2013 (14) en el cual se estandarizo el cultivo de esta línea celular con el fin de ser utilizadas en protocolos de infección con $C T$, encontrando que es necesario cultivarlas en medio como el DMEM-F12. En este estudio se realizó la comparación de las características de crecimiento de las células HeLa y las células HEp-2, Figura 7 / Tabla 2, lográndose obtener un mejor crecimiento de la línea celular HeLa ya que consigue la confluencia necesaria en un rango de tiempo menor que las HEp-2 y con menores exigencias respecto a medio y concentración de L-glutamina.

\section{Referencias}

1. Bastidas RJ, Elwell CA, Engel JN, Valdivia RH. Chlamydial intracellular survival strategies. Cold Spring Harb Perspect Med. 2013 May 1; 3(5). http://perspectivesinmedicine.cshlp. org/content/3/5/a010256.long. (ultimo acceso 17 de junio de 2014)

2. Jorgensen I, Bednar MM, Amin V, Davis BK, Ting JP, McCafferty DG, Valdivia RH. The Chlamydia protease CPAF regulates host and bacterial proteins to maintain pathogen vacuole integrity and promote virulence. Cell Host Microbe. $2011 \mathrm{Jul} 21$; 10(1): 21-32. Disponible en: http://www. ncbi.nlm.nih.gov/pmc/articles/PMC3147293/ (ultimo acceso 17 de junio 2014)

3. Choroszy-Król IC, Frej-Mądrzak M, Jama-Kmiecik A, Bober T, Jolanta Sarowska J. Characteristics of the Chlamydia trachomatis species - immunopathology and infections. Adv Clin Exp Med. 2012 Nov;21(6):799-808.

4. Joyce A Ibana, Leann Myers, Constance Porretta, Maria Lewis, Stephanie N Taylor, David H Martin and Alison J Quayle. The major CD8 T cell effector memory subset in the normal and Chlamydia trachomatis-infected human endocervix is low in perforin. BMC Immunology. 2012 Dec 12: 13. http://www.biomedcentral.com/1471-2172/13/66. (ultimo acceso 05 de octubre de 2014)

5. Clotilde Vallejos M, Guerra Ml Á, López Villegas M R, J. Valdez García A, Pría Kasusky P. Cérvico-vaginitis por Chlamydia trachomatis en mujeres atendidas en un hospital de Acatlán de Osorio, Puebla. Enf Inf Microbiol. 2010 abr-jun. 30(2): 49-52.
Disponible en : http://www.medigraphic.com/pdfs/micro/ei2010/ei102c.pdf (ultimo acceso 06 de septiembre 2014)

6. Martínez M. A, Diomedi Alexis P, Kogan A.Ricardo y Borie P C. Taxonomía e importancia clínica de las nuevas familias del orden Chlamydiales. Rev. chil. infectol. 2001; 18(3): 203-211. Disponible en: http://www.scielo.cl/scielo.php?script=sci_ arttext\&pid=S0716-10182001000300007 \&lng=es. http:// dx.doi.org/10.4067/S0716-10182001000300007. (Ultimo acceso 15 de julio 2014)

7. Health topics Trachoma and Epidemiology. Organización Mundial de la Salud. 2013. Disponible en: http://search.who. int/search?q=chlamydia+trachomatis+epidemiology\&ie=utf8 \&client=_en_r\&proxystylesheet=_en_r\&output=xml_no_dt d\&oe=utf\&\&getfields=doctype\&site=amro_alia. (ultimo acceso 2013 septiembre 15 )

8. Molano M, Weiderpass E, Posso H, Morre SA, Ronderos M, Franceschi S, et al; HPV Study Group. Prevalence and determinants of Chlamydia trachomatis infections in women from Bogota, Colombia. Sex Transm Infect. 2003. 79:474-478. Disponible en: http://www.ncbi.nlm.nih.gov/pmc/articles/ PMC1744784/ (ultimo acceso 2 de mayo 2014)

9. Cheng W, Shivshankar P, Zhong Y, Chen D, Li Z, Zhong G. Intracellular interleukin-1alpha mediates interleukin-8 production induced by Chlamydia trachomatis infection via a mechanism independent of type I interleukin-1 receptor. Infection and immunity. 2008:76(3):942-51 Disponible en: http://www.ncbi.nlm.nih.gov/pmc /articles/PMC2258806/ (ultimo acceso 2 de mayo de 2014)

10. Marti H, Koschwanez M, Pesch T, Blenn C, Borel N. Waterfiltered infrared an irradiation in combination with visible light inhibits acute chlamydial infection. PloS one. 2014: 9(7): http://journals.plos.org/plosone/article?id=10.1371/journal. pone.0102239 (ultimo acceso 16 de julio 2014)

11. HeLa: Las primeras células humanas inmortales. NeoTeo. 2009: Disponible en: http://www.neoteo.com/hela-las-primeras-celulashumanas-inmortales-15154/. (ultimo acceso 05 de octubre 2013)

12. DRA. Anticuerpos Antinucleares (ANA) en células Hep-2. Sociedad chilena de alergia e inmunologia: Disponible en: http:// www.scai.cl/node/28 (ultimo acceso 08 de diciembre 2014)

13. Invitrogen G. Cell culture basics. Life technologies. 2014; Disponible en: http://www.lifetechnologies.com/gibco-cellculture-basics (ultimo acceso 14 de octubre 2014).

14. Adriana Paola Juntinico Shubach, Johanna Nathaly Manrique Chacón. Estandarización del cultivo celular de HEp-2 y las perspectivas para evaluar péptidos antimicrobianos en células infectadas con Chlamydia trachomatis Colombia. Tesis de grado. Universidad colegio mayor de Cundinamarca; 2013.

15. Innovación Científica en Biología Molecular y Celular. Cientifica Senna S.A. de C.V. 2014: Disponible en: http://www. cientificasenna.com/ index.php? modulo=catalogo $\&$ accion $=$ ar ticulo\&id=1552 (ultimo acceso 12 de julio 2014)

16. Escobar M Lina maría, Morantes Sandra, Cordero Claudia P., Aristizábal Fabio A. Implementación de estrategias in vitro para evaluar la funcionalidad de un suero fetal bovino colombiano. Rev. colomb. cienc. quim. farm. 2011. 40(2): 201-221. Disponible en: http://www.scielo.org.co/ scielo.php?script=sci_ arttext\&pid=S0034- 74182011000200005\&lng=en (ultimo acceso 01 de agosto de 2014) 
17. Obtención de un frotis sanguíneo y tinción de giemsa. Wikifisiologia. 2014 :Disponible en: http://wiki.fisiologia.me/images/4/49/Cris2.pdf. (ultimo acceso 08 de agosto 2014)

18. Diagnostica I. coloracion de giemsa. IHR diagnostica. 2007. Disponible en: http://www.ihrdiagnostica.com/tecnicas/pdf/ ColoracionGIEMSAv2.pdf. (ultimo acceso 07 de junio 2014)

19. Stoker MGP, Smith, K. M. \& Ross, R. W. Electron Microscope Studies of HeLa Cells Infected with Herpes Virus. J gen Microbiol.1958.19: 244-249 Disponible en: http://mic. sgmjournals.org/ content/journal/micro/ 10.1099/0022128719-2-244 (ultimo acceso 2 de junio de 2014)

20. James E. Darnell J. Adsorption and maturation of poliovirus singly and multiply infected HeLa cells. Jorurnal of experimental medicine. 1958:107 (5):633-641 Disponible en: http://www.ncbi.nlm.nih.gov/pmc/articles /PMC2136847/ (ultimo acceso 25 agosto 2014).

21. Minato N, Takeda A, Kano S, Takaku F. Studies of the Functions of Natural Killer-Interferon System in Patients with Sjogren Syndrome. the journal of clinical investigation. 1982. 69(3), 581-588. Disponible en: http://www.ncbi.nlm.nih. gov/pmc/articles/PMC371014/ (ultimo acceso 21 de mayo de 2014)

22. Modulevsky DJ, Lefebvre C, Haase K, Al-Rekabi Z, Pelling AE. Apple derived cellulose scaffolds for 3D mammalian cell culture. PloS one. 2014;9(5): Disponible en: http://journals. plos.org/plosone/ article?id=10.1371/journal.pone.0097835 (ultimo acceso 21 de julio 2014) .

23. Tsugeno Y, Sato F, Muragaki Y, Kato Y. Cell culture of human gingival fibroblasts, oral cancer cells and mesothelioma cells with serum-free media, STK1 and STK2. Biomedical reports. 2014;2(5):644-8. Disponible en: http://www.ncbi.nlm.nih. gov/pmc/articles/PMC4106576/ (Ultimo acceso 24 de julio 2014)

24. Hegde S, Spergser J, Brunthaler R, Rosengarten R, ChopraDewasthaly $R$. In vitro and in vivo cell invasion and systemic spreading of Mycoplasma agalactiae in the sheep infection model. International journal of medical microbiology : IJMM. 2014. 304(8), 1024-1031. Disponible en: http://www.ncbi. nlm.nih.gov/pmc/articles/PMC4282308/ (Ultimo acceso 19 de agosto 2014)

25. Wong YH, Tan WY, Tan CP, Long K, Nyam KL. Cytotoxic activity of kenaf (Hibiscus cannabinus L.) seed extract and oil against human cancer cell lines. Asian Pacific journal of tropical biomedicine. 2014;4(Suppl 1). Disponible en: http://www. sciencedirect.com/science/article/pii/S2221169115303191 ( u ltimo acceso 04 de septiembre 2014).

26. Hayashi-Takanaka Y, Stasevich TJ, Kurumizaka H, Nozaki N, Kimura H. Evaluation of Chemical Fluorescent Dyes as a Protein Conjugation Partner for Live Cell Imaging. PloS one. 2014; 9(9). Disponible en: http://journals.plos.org/plosone/ article?id=10.1371/journal.pone.0106271(ultimo acceso 10 de octubre de 2014).

27. Thiede B, Koehler CJ, Strozynski M, Treumann A, Stein R, Zimny-Arndt U, Schmid M, Jungblut PR.. High Resolution Quantitative Proteomics of HeLa Cells Protein Species Using Stable Isotope Labeling with Amino Acids in Cell Culture(SILAC), Two-Dimensional Gel Electrophoresis(2DE) and Nano-Liquid Chromatograpohy Coupled to an LTQ-OrbitrapMass Spectrometer. Mol Cell Proteomics. 2013. 12(2), 529-538. Disponible en: http://www.mcponline.org/content/12/2/529.long (ultimo acceso 15 de julio de 2014)

28. Chen TR. Re-evaluation of HeLa, HeLa S3, and HEp-2 karyotypes. Cytogenetics and cell genetics. 1988;48(1):19-24.

29. Pajaniradje S, Mohankumar K, Pamidimukkala R, Subramanian S, Rajagopalan R. Antiproliferative and apoptotic effects of Sesbania grandiflora leaves in human cancer cells. BioMed research international. 2014; Disponible en: http://www.ncbi. nlm.nih.gov/pmc/articles/PMC4053233/ (ultimo acceso 03 de septiembre de 2014)

30. Arevalo-Pinzon G, Curtidor H, Munoz M, Patarroyo MA, Patarroyo ME. Synthetic peptides from two Pf sporozoite invasion-associated proteins specifically interact with HeLa and HepG2 cells. Peptides. 2011;32(9):1902-8. Disponible en: http://www.sciencedirect.com/ science/article/pii/ S0196978111003275 (ultimo acceso 21 de agosto 2014)

31. Himeda T, Hosomi T, Okuwa T, Muraki Y, Ohara Y. Saffold virus type 3 (SAFV-3) persists in HeLa cells. PloS one. 2013;8(1). Disponible en: http://journals.plos.org/plosone/ article? id=10.1371/journal.pone.0053194 (ultimo acceso 28 de septiembre 2014)

32. Li L, Wang L, Xiao R, Zhu G, Li Y, Liu C, et al. The invasion of tobacco mosaic virus RNA induces endoplasmic reticulum stress-related autophagy in HeLa cells. Bioscience reports. 2012;32(2):171-86. 